\title{
Vom Deponierückbau bis zum landfill mining - eine Synthese internationaler Untersuchungen
}

Zusammenfassung: Der Deponierückbau stellt eine Möglichkeit dar Gefahren der Grund- und Oberflächenwasserverschmutzung abzuwenden. Die Preissteigerungen für Rohstoffe in den Jahren 2003 bis 2008 hatten in Deutschland die Diskussion Deponien zum Zwecke der Wertstoffgewinnung rückzubauen aufleben lassen. In diesem Falle sind die stoffliche Zusammensetzung der Deponie und die regionale Verwertungsinfrastruktur für die wirtschaftliche Machbarkeit entscheidend. Von großer Bedeutung ist der Anteil an Metallen und heizwertreicher Materialien, wie Kunststoffe und Holz, aber auch zu entsorgende gefährliche Abfälle und nicht verwertbare Fraktionen wie beispielsweise mineralische Stoffe. Große Mengen an mineralischen Bestandteilen stellen hinsichtlich der Verwertung ein Problem dar. Je nach Entwicklung der Verwertungs- und Beseitigungsinfrastruktur können erhebliche Transportwege und damit verbundene Umweltauswirkungen entstehen. Die bisherigen Erfahrungen zeigten, daß die stoffliche Zusammensetzung von Deponien und Rohstoffpreise einen Rückbau mit dem Ziel der Wertstoffrückgewinnung in absehbarer Zeit meist nicht wirtschaftlich erscheinen läßt. Jedoch kann anhand der betrachteten Fallbeispiele davon ausgegangen werden, daß ein Deponierückbau zur Sicherung der Trinkwasserversorgung nicht nur im Sinne des Gesundheitsschutzes sondern auch wirtschaftlich vertretbar ist.

From landfill-site remediation to landfill mining - a synthesis of international studies

Summary: Remediation of landfill sites is a possibility for preventing the pollution risk for ground and surface water. Increasing raw-materials prices in the years 2003 to 2008 have triggered discussions in Germany regarding the possibility of excavating landfill sites with the aim of recovering recyclable material. The main factors in terms of economic feasibility are in this case the substance composition of a landfill site and the regional recycling infrastructure. Points of great importance are the

\begin{tabular}{ll}
$\begin{array}{l}\text { TABELLE 1 } \\
\text { Headline }\end{array}$ & \\
Deponie & Grund \\
\hline Heckenweiher/Deutschland & Gefahrenabwehr (Grund- und Oberflächenwasser) \\
\hline Holzg'wandenweg/Deutschland & Gefahrenabwehr (Grund- und Oberflächenwasser) \\
\hline Tiefenbach/Deutschland & Gefahrenabwehr (Oberflächenwasser) \\
\hline Jagerberg/Deutschland & Gefahrenabwehr (Grundwasser) \\
\hline Dougal/Kanada & Gefahrenabwehr (Grundwasser) \\
\hline Rennerod/Deutschland & Gefahrenabwehr (Grundwasser) \\
\hline Scheffau/Deutschland & Gefahrenabwehr (Stabilität) \\
\hline Burghof/Deutschland & $\begin{array}{l}\text { Gewinnung von Deponievolumen, Prüfung der technischen und } \\
\text { wirtschaftlichen Machbarkeit des Deponierückbaus }\end{array}$ \\
\hline Naples/USA & Prüfung der technischen und wirtschaftlichen Machbarkeit \\
\hline Edinburg/USA & Prüfung der technischen und wirtschaftlichen Machbarkeit \\
\hline Frey Farm/USA & Gewinnung von heizwertreicher Fraktion zur Auslastung der MVA \\
\hline Thompson/USA & Gewinnung von Deponievolumen \\
\hline Guhagoda/Sri Lanka & Gewinnung von Deponievolumen \\
\hline Elbisgraben/Schweiz & Gewinnung von Deponievolumen und Metallgewinnung \\
\hline Sharjah/UAE & Stadtwachstum \\
\hline & \\
\hline
\end{tabular}

concentrations of metals and materials of high calorific value such as synthetics and wood, as well as expensive disposals of hazardous waste and non-recyclable fractions such as mineral substances. Large proportions of mineral constituents pose recycling problems. Substantial transport distances including the environmental impact may be involved, depending on the network and development of the recycling infrastructure. Experience gathered so far has shown that the substance composition of landfills and the present raw-material prices are unlikely to make landfill mining, with the aim of material recycling, appear economical in the foreseeable future. But the results of our case studies also show that landfill-site remediation intended to safeguard a reliable drinking-water supply is not only useful in terms of health protection but is also economically justified.

\section{Allgemein}

Der Betrieb einer Deponie geht lange über die Zeit der Ablagerung hinaus, ohne Ein- nahmen aus der Anlieferung. Deponien sind mit langfristigen Umweltauswirkungen, Nachsorgekosten und -zeiträumen verbunden. $\mathrm{Zu}$ den Problematiken zählen Emissionen klimarelevanter Deponiegase, Grundwasserverunreinigungen durch Sickerwässer, Standorte in Hochwasserbereichen, Nachsorgeverantwortung, Rechtsnachfolge, neue Umweltgesetzgebungen, zunehmende Flächeninanspruchnahme, begrenzte Nutzungsmöglichkeiten etc.Ziel des Deponieabschlusses ist die Minimierung von Gefahren für Mensch und Umwelt durch Abkapselung der Deponie und die Wiedereingliederung in das Landschaftsbild durch Modellierungs- und Rekultivierungsmaßnahmen.

Neue Ideen der Folgenutzung für Deponien wie Energie- und Recyclingparks (z.B. Windräder, Photovoltaikanlagen, Energiepflanzenanbau, Aufbereitungsanlagen etc.), wurden in den letzten Jahren umgesetzt. Der Deponierückbau, sofern die Wertstoffgewinnung im Vordergrund steht auch landfill mining genannt, hat wegen der hohen Rohstoffpreise in den letzten Jahren wieder mehr Aufmerksamkeit erhalten (Fricke 2009, Mocker et al. 
2009). Die Geschichte von Deponierückbaumaßnahmen reicht bis in die 50er Jahre zurück. Die Gründe bisheriger Rückbauten waren sehr unterschiedlich, in den seltensten Fällen jedoch zur Wertstoffgewinnung (Tab. 1). Die Tendenz zeigt, dass in ländlichen Gebieten Grund- und Oberflächenwasserschutz und in den städtischen Gebieten Platzmangel sowie mangelndes Deponievolumen ausschlaggebende Gründe für den Rückbau waren.

In Bayern wurde mit In-Kraft-Treten des Gesetzes zur Änderung des Bayerischen Bodenschutzgesetzes und des Bayerischen Abfallwirtschaftgesetzes zum 01.05.2006 ein neuer Unterstützungsfonds eingerichtet. Dort können kreisangehörige Gemeinden finanzielle Mittel für die Erkundung und Sanierung ihrer ehemaligen Hausmülldeponien beantragen. Seitdem wurden sechs Deponien komplett zurückgebaut, da Grund- und Oberflächenwasser kontaminiert waren oder die unmittelbare Gefahr der Kontamination gegeben war. Die Deponien beinhalteten Hausmüll und Bauschutt. Hingegen wiesen bisherige Rückbauprojekte in Österreich, wie beispielsweise die Helene Berger Deponie, spezielle stoffliche Zusammensetzungen auf, und die Trennung und Entsorgung des Gewerbe- und Industriemülls stellte sich aufwendig dar (UBA 2003). Die Änderungen der Technischen Anleitung Siedlungsabfall (TASi) in Deutschland zum 01.06. 2005 erlaubten keine Umlagerung des Deponats mit unvorbehandelten Siedlungsabfällen auf andere Deponien. Somit war eine Trennung und stoffgerechte Verwertung der ausgegrabenen Materialien notwendig.

Im Folgenden sollen Erfahrungen und charakteristische Kennzahlen zu Trennverfahren, Wertstoffpotenzial, Stoffströmen, Verwertung, Kosten und Nutzen bayerischer und internationaler Deponierückbauprojekte betrachtet werden.

\section{Deponierückbau}

Ein Deponierückbau beginnt in der Regel mit Voruntersuchungen wie Bohrungen oder auch Grundwassermessungen und schließt mit Geländemodellierungs- und Rekultivierungsmaßnahmen ab. Die Voruntersuchungen zielen in erster Linie darauf ab, die stoffliche Zusammensetzung, Kubaturen und die räumlichen Ausmaße sowie in den entwickelten Ländern auch die Gefährdungspotenziale und Rechtsverhältnisse zu bestimmen. In Bayern ist dies ein mehrstufiger Prozess, der aus einer historischen Erkundung, einer orientierenden

Untersuchung, einer Detailuntersuchung und einer Sanierungsuntersuchung oder -planung besteht.

Die anschließende Baustelleneinrichtung ist zum Teil mit erheblichem Aufwand verbunden, da Entgasungsanlagen und Insitu-Belüftungen, Bereiche für stark kontaminiertes Aushubmaterial, Grundwasserreinigungsanlagen, Schwarz-/ Weißbereiche (Trennung von Arbeits-

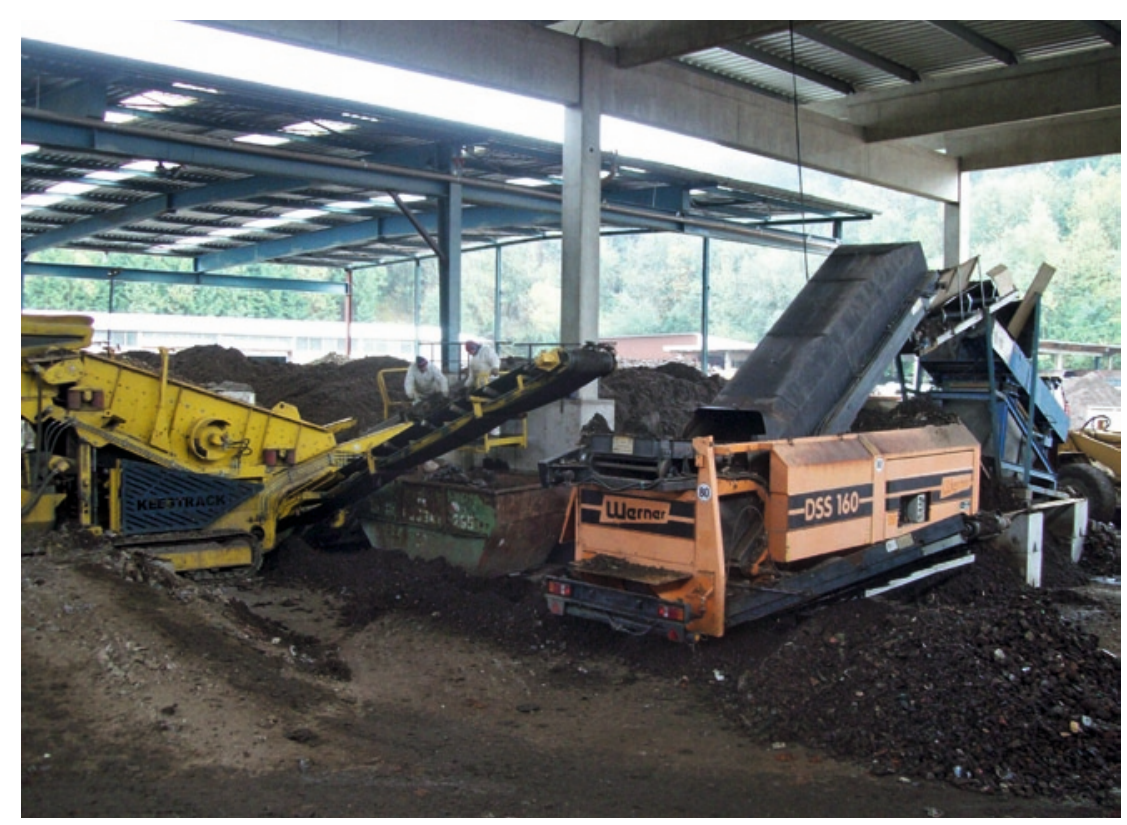

Abb. 1: Trennverfahren auf dem Gelände der AKR. V.I.n.r. Schwerlastsiebanlage mit manueller Nachsortierung, Schwerefalle und Spannwellensieb.

bereichen zur Vermeidung der Schadstoffverschleppung), Sortieranlagen oder gar eine Seilbahn wie im Falle des Rückbaus der Deponie Scheffau in Bayern, eingerichtet werden müssen.

Der Rückbau erfolgt entweder vollständig, d.h. das gesamte Material wird ausgehoben und abtransportiert, oder nur teilweise, indem wirtschaftlich interessante bzw. störende Fraktionen abgetrennt und das restliche Material wieder eingebaut werden. Für den ausschließlichen Deponievolumen zu gewinn reichen die Zerkleinerung voluminöser Fraktionen und der Wiedereinbau des gesamten Materials aus. Die Sortierung erfolgt bevorzugt vor Ort, vorausgesetzt für die Sortieranlagen gibt es ausreichend Platz, die Behörden stimmen zu und die Störung der Anrainer bleibt erträglich.

Die abschließende Geländemodellierung erfolgt teilweise mit Primärrohstoffen wie Schotter und Kies sowie einer Rekultivierungsschicht. Der eigentliche Rückbau nimmt bei kleinen Deponien zeitlich nur einen Bruchteil in diesem Prozessverlauf ein.

\section{Trennverfahren}

Die Geräte bei Rückbaumaßnahmen reichten von Schaufel und einfachen "Gartensieben“ bei einer Deponie in Indien (Scheuer et al. 1997) bis zu infrarotsensorgestützten Systemen bei Deponien in Europa. Weltweit fanden am meisten Siebe jeglicher Art, insbesondere Trommelsiebe, Verwendung. Bereits in den 


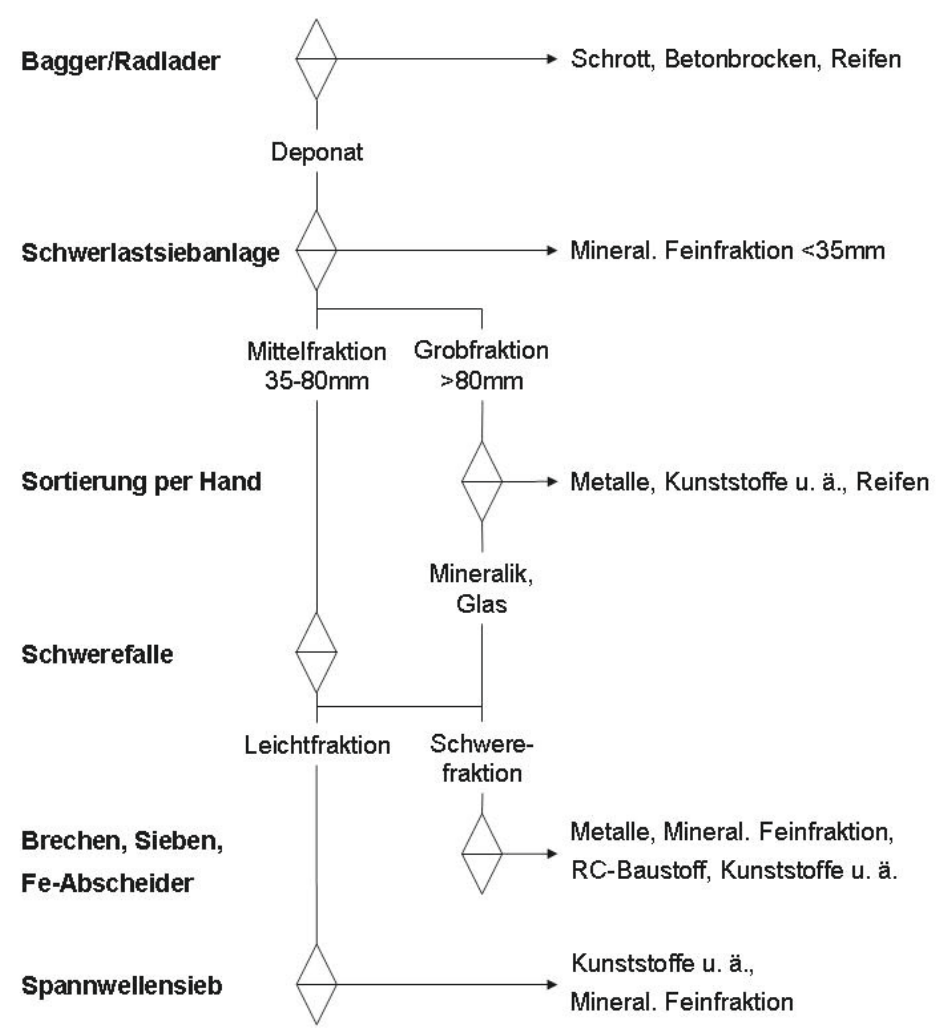

Abb. 2: Trennprozessschritte und Fraktionen bei der Deponie Jagerbergl durch die AKR. gelagert wurde, durchlief mehrere Trennverfahren ( $s . A b b .2)$. Auf der Deponie und dem Betriebsgelände fand erst eine Grobabtrennung großer Gegenstände wie Schrott, Betonbrocken, Holz und Reifen, mittels Bagger und Radlader statt. Das verbliebene Deponat durchlief anschließend eine Schwerlastsiebanlage (Stangensizer und Schwingsieb) mit drei Trennschnitten $\quad(0-35 \mathrm{~mm} / 35-80 \mathrm{~mm} /$ $>80 \mathrm{~mm}$ ). Ziel war es, eine mineralische Feinfraktion $(<35 \mathrm{~mm})$ mit weniger als $5 \%$ Fremdanteil zu erhalten, um diese nach Deponieverordnung verwerten zu können (Zobel et al. 2010). Die gewon- nene Mittelfraktion (35-80 mm) gelangte zur weiteren Trennung in eine Schwerefalle. Die Grobfraktion unterlief anschließend eine händische Trennung (Metalle, Reifen, Mineralik, Kunststoffe u.ä.). In der Mineralik der Grobfraktion befand sich ein bemerkenswerter Anteil an Glasflaschen. Folgende Materialien stellten für den Betrieb der Schwerlastsiebanlage ein Problem dar:

- Strumpfhosen, die sich verwickelten und verhängten

- Folien, die Sieböffnungen verdeckten

- Gesteinsbrocken, die der Anlage Schäden zufügten

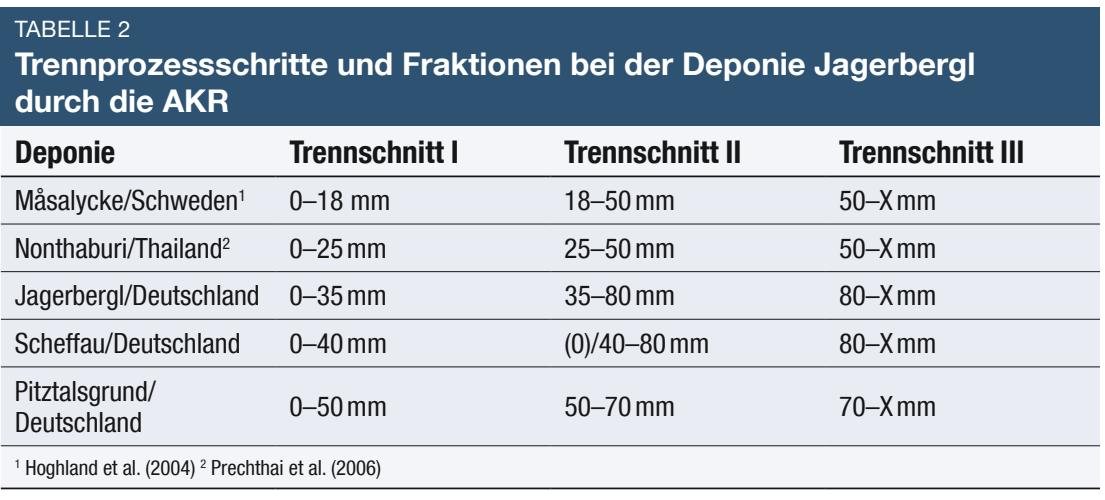

Bei der Schwerefalle entstanden eine Fraktion mit Mineralik und schwereren Plastikgegenständen sowie eine Leichtfraktion mit Sand, Schluff, Textilien und Kunststoffen. Die Mineralik-Plastik-Fraktion wurde anschließend in einer Brech- und Siebanlage aufgetrennt. Die Leichtfraktion gelangte direkt in eine Spannwellensiebanlage, bei der eine Trennung in eine mineralische Feinfraktion und eine Kunststoff-Textil-Fraktion erfolgte.

Die Durchführung von zwei Trennschnitten erwies sich in vielen Projekten von Vorteil. Die Sieböffnungen lagen zwischen 18-50 mm für den Feinanteil und zwischen $35-80 \mathrm{~mm}$ für den Grobanteil. Grundsätzlich variiert der optimale Trennschnitt von Deponie zu Deponie, aber auch das wechselnde Deponat einer Deponie kann eine Anpassung der Sieböffnungen während des Rückbaus erfordern. Tabelle 1 gibt einen Überblicküber die Trennschnitte internationaler Deponierückbauten.

Von Vorteil erwiesen sich bei den bayerischen Deponierückbauprojekten Stangensizer oder auch Sternsiebe. Als erstes Trennverfahren eingesetzt, ermöglichten sie es bindige Deponate aufzulockern. Bei Trommelsieben verdeckten bindige und folienhaltige Materialien leicht die Öffnungen. Sternsiebe zeigten jedoch schneller Verschleißerscheinungen, sofern das Material Gesteinsbrocken enthielt. Der Einsatz von Windsichtern wie einfache Heugebläse, Schneekanonen oder konventionelle Sichter war nur von mäßigem Erfolg. Probleme stellten unter anderem das Ausblasen von feiner Mineralik oder auch zu erwartende Geräteschäden durch grobe Mineralik dar.

\section{Wertstoffe}

Die stoffliche Zusammensetzung von Deponien ist von großer Bedeutung, wenn die Vermarktung der Wertstoffe im Vor-

\section{TABELLE 4}

Brennwerte der Leichtfraktionen ausgewählter Deponien

\begin{tabular}{ll}
\hline Deponie & Brennwert in $\mathbf{~ J / k g}$ \\
\hline Edinburg/USA & $7.160(3,080 \mathrm{Btu} / \mathrm{lb})$ \\
\hline Filborna/Schweden ${ }^{2}$ & $6.900-7.900$ \\
\hline Naples/USA ${ }^{3}$ & $14.421(6.200 \mathrm{Btu} / \mathrm{lb})$ \\
\hline Deponie $\mathrm{A}^{4}$ & $18.000-20.000$ \\
\hline Deponie B & $20.000-22.000$ \\
\hline${ }^{1}$ EPA (1997) ${ }^{2}$ Hoghland et al. (2004) \\
${ }^{3} \mathrm{EPA}(1993){ }^{4}$ Rettenberger (2009) \\
\hline
\end{tabular}




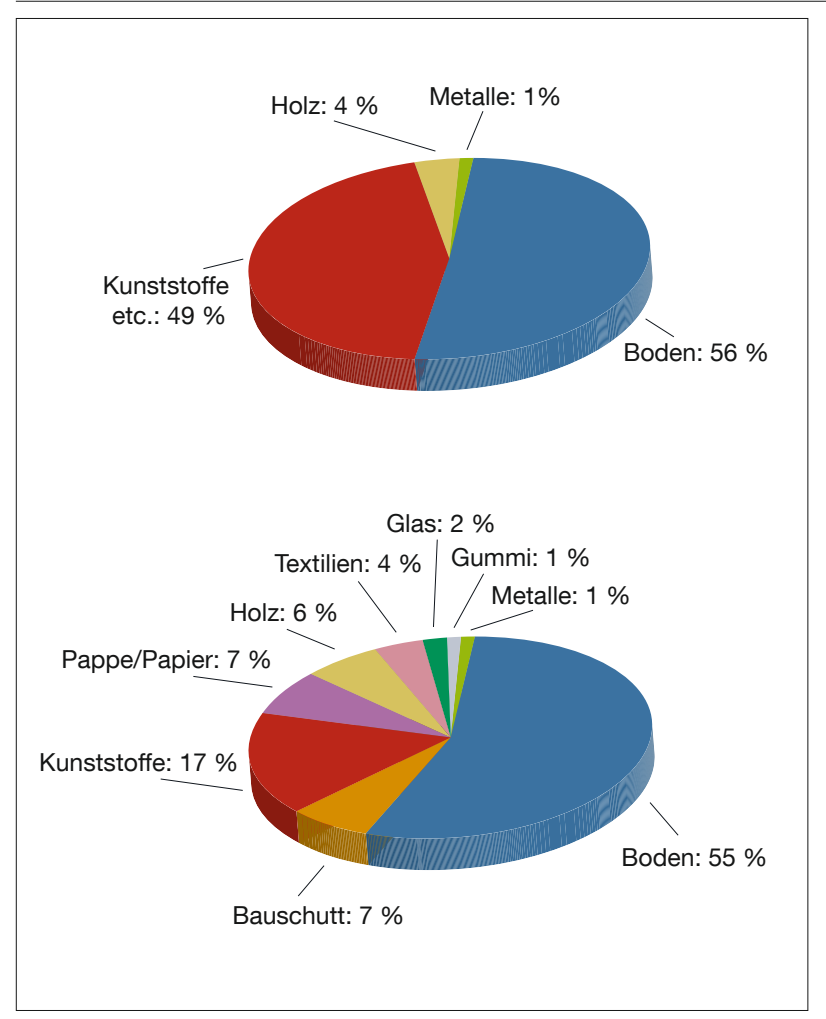

Abb. 3: Verschiedene Wertstofffraktionen der manuellen Voruntersuchung (unten) und der maschinellen Trennung (oben) (modifiziert nach Koch 2005 und Göschl 2006).

dergrund steht. Bei der Betrachtung des Wertstoffpotenzials einer Deponie muss jedoch zwischen theoretischem Potenzial und den tatsächlich trennbaren Wertstoffmengen unterschieden werden. Ersteres wird in der Regel in kleinen Versuchen per händischer Trennung er- mittelt. Letztere stellen die Mengen der Wertstoffe dar, die durch großmaschinelle automatisierte Trennung erreicht werden. Ein deutlicher Unterschied zeigte sich beim Deponierückbau in Sharjah (Vereinigte Arabische Emirate). Die Vorsortierversuche führten zu neun verschiedene Fraktionen (Koch 2005) im Vergleich zu vier gewonnen Fraktionen beim Rückbau (Göschl 2006) (s. Abb. 3). Die unterschiedlichen Mengenanteile sind in diesem Fall vor allem auf die Verwendung von $\mathrm{Ge}$ wichtsprozenten bei der Voruntersuchung und Volumenprozenten bei der Rückbaumengenbilanz zurückzuführen sowie auf die Trocknung des ausgegrabenen Holzes.

Die stoffliche Zusammensetzung hängt primär von den abgelagerten Materialien und dem Grad der Zersetzung ab. Diese beiden Faktoren werden maßgeblich von wirtschaftlichen und sozialen Entwicklungen, dem Einzugsbereich (Stadt-Land), der Vorbehandlung des Materials, der Wertstoffsammlung während des Deponiebetriebs und klimatischen sowie hydrogeologischen Bedingungen beeinflusst.

Die Auswertung internationaler Rückbauprojekte zeigt, dass meistens die Mineralik den größten Anteil, Kunststoffe und ähnliches einen Wert zwischen 15 und $50 \%$ und Metalle sowie Holz weit unter $10 \%$ aufweisen können (Tab. 3).

Für die energetisch zu verwertenden Leichtfraktionen sind die Brennwerte, aber auch der entstehende Aschegehalt von großer Bedeutung. Bei bisherigen Untersuchungen lagen die Brennwerte zwischen 7.160 und $22.000 \mathrm{~kJ} / \mathrm{kg}$ (s. Tab. 4).

\section{Stoffströme und Verwertung}

Die Stoffströme und die Verwertung hängen primär vom Markt und gesetzlichen Vorgaben ab. Während bei einer Deponie in Indien (Scheuer et al.) und Sri Lanka (Werellagama et al. 2007) die Gewinnung organischer Materialien für die Kompostherstellung im Vordergrund standen, wurde in den Industrieländern die Gewinnung von Metallen und heizwertreichen Fraktionen, bei gleichzeitig möglichst geringem Mineralikanteil angestrebt. In Deutschland ist die Nachfrage für Recycling-Baustoffe sehr gering. Folglich werden die mineralischen Fraktionen überwiegend in Deponien verwertet. Auch für die Verwertung der angefallenen $18.000 \mathrm{~m}^{3}$ Holz beim Deponierückbau in Sharjah

\section{TABELLE 3}

Mengen der bedeutenden Fraktionen internationaler Deponierückbauten

\begin{tabular}{|c|c|c|c|c|c|c|c|c|c|c|c|}
\hline Deponie & 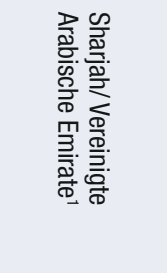 & 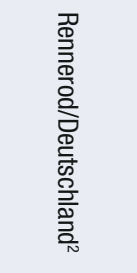 & 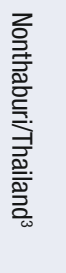 & 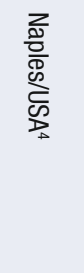 & 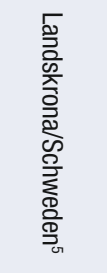 & 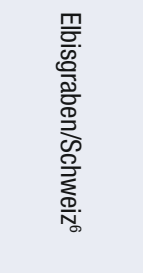 & 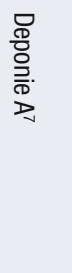 & 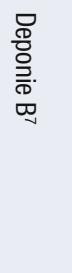 & 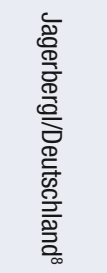 & 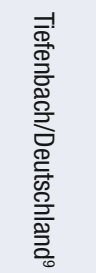 & 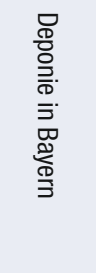 \\
\hline Volumen/Masse & $1.730 .525 \mathrm{~m}^{3 \mathrm{a}}$ & $785.000 \mathrm{~m}^{3}$ & b & $292 \mathrm{t}^{\mathrm{b}}$ & $1.800 \mathrm{t}^{\mathrm{c}}$ & $\sim 180.000 \mathrm{t}^{\mathrm{d}}$ & e & e & $18.000 \mathrm{t}$ & $9.300 t$ & $7.100 t$ \\
\hline Mineralik \% & 56 & & 31,8 & 60 & 60 & & 82,7 & 55,2 & 94,4 & 98,9 & 92,1 \\
\hline Kunststoffe etc.. ${ }^{9} \%$ & 48,8 & 21,8 & 52,2 & 2 & 30 & 0 & 14,3 & 35,4 & 3,3 & 0,1 & 6,1 \\
\hline Metalle \% & 4,1 & 4,1 & 3,0 & 2 & $2-3$ & 4 & $1,2^{f}$ & $3,5^{f}$ & 1,1 & 0,7 & 0,3 \\
\hline Holz \% & 1,1 & 5,3 & 9,0 & & 1 & 0 & 1,8 & 5,8 & & 0,3 & 0,1 \\
\hline
\end{tabular}

1)Göschl (2006); 2)Kreisverwaltung des Westerwaldkreises (2006); 3)Prechthai et al. (2006); 4)EPA (1993); 5)Bergstedt (2008); 6)Fitze et al. (2008); 7)Rettenberger (2009); 8)Zobel et al. (2010); 9)Danzer et al. (2008) a) Damaliger Stand des Rückbaus, Deponiegesamtvolumen 6,4 Mio. m3

b) Pilotuntersuchung

c) Pilotuntersuchung, Deponiegesamtvolumen $150.000-300.000 \mathrm{~m} 3$

d) Deponie ausschließlich mit Müllverbrennungsschlacken

e) Keine Angaben zur Größe oder Volumen

e) Keine Angaben zur Grientien Eisen

g) In der Regel Gemisch aus Kunststoffen, Textilien, Papier und Papp 


\begin{tabular}{|c|c|}
\hline Stoffgruppe & Verwertung \\
\hline Grobmineralik & RC-Baustoff im Straßen- und Wegebau, Deponiebaumaßnahmen \\
\hline Feinmineralik & Deponiebaumaßnahmen \\
\hline $\begin{array}{l}\text { Kunststoffe, Textilien, } \\
\text { Papier, Gummi, etc. }\end{array}$ & Energetische Verwertung \\
\hline Reifen & Energetische Verwertung, v.a. in der Zementindustrie \\
\hline Metalle & Stoffliche Verwertung \\
\hline Holz & Energetische Verwertung \\
\hline Organik & Kompost- und Biogasherstellung \\
\hline Glas & Stoffliche Verwertung, Deponiebaumaßnahmen \\
\hline
\end{tabular}

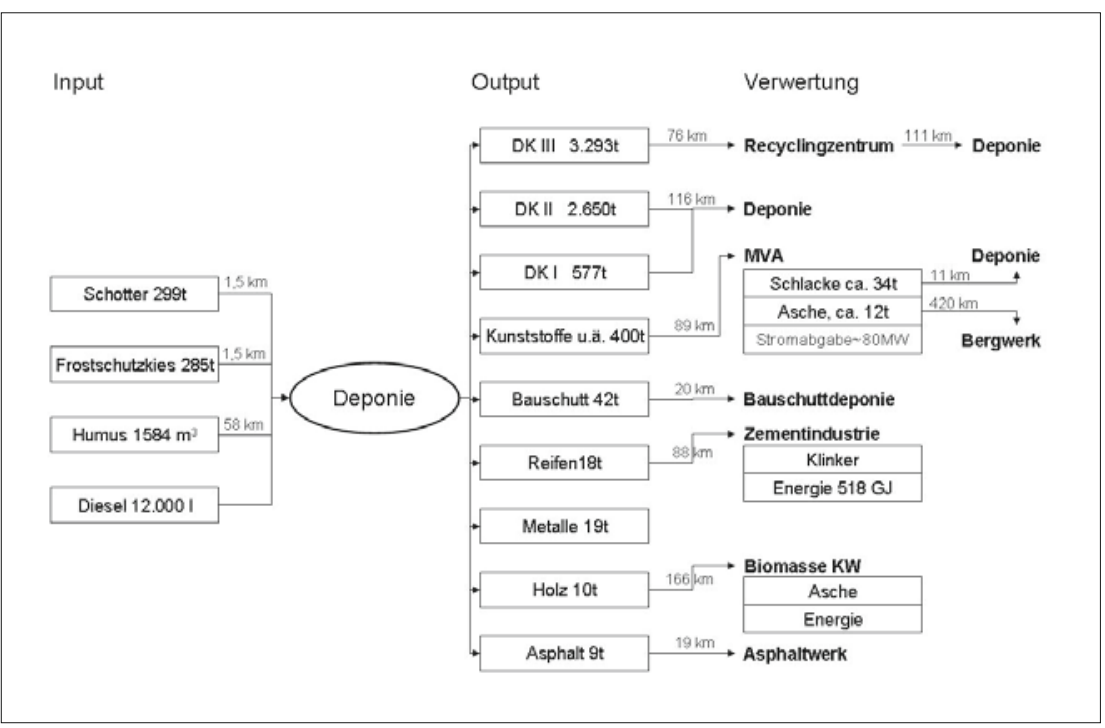

Abb. 4: Stoffstromflüsse mit Mengen und Entfernungen bei einer Deponie in Bayern - Energie (alle Transportdistanzen einfach).

\section{TABELLE 6}

Transportkilometer und geschätzter Dieselverbrauch für Transport, Ausgrabung und Vorsortierung bei einem Deponierückbau in Bayern (alle Transportdistanzen einfach)

\begin{tabular}{lrcc} 
Material & Transporte in $\mathbf{~ m m}$ & $\begin{array}{l}\text { Dieselverbrauch } \\
\text { ca. I/100 } \mathbf{~ m m}\end{array}$ & $\begin{array}{c}\text { Gesamtdiesel- } \\
\text { verbrauch in I }\end{array}$ \\
\hline DK I & 2.436 & 35 & 853 \\
\hline DK II & 11.136 & 35 & 3.898 \\
\hline DK III & 22.253 & 35 & 7.789 \\
\hline Kunststoffe & 3.169 & 30 & 951 \\
\hline Altholz & 166 & 35 & 58 \\
\hline Bauschutt, z.T. bewehrt & 100 & 35 & 35 \\
\hline Altreifen & 88 & 35 & 31 \\
\hline Asphalt & 19 & 35 & $\mathbf{1 3 . 6 2 0}$ \\
\hline Gesamtkilometer & $\mathbf{3 9 . 3 6 7}$ & Gesamtverbrauch & $\mathbf{1 1 . 8 7 5}$ \\
\hline Gesamttonnage & $\mathbf{7 . 0 8 0}$ & $\begin{array}{l}\text { Ausgrabung und } \\
\text { Vorsortierung }\end{array}$ & $\mathbf{2 5 . 4 9 5}$ \\
\hline & & Gesamtsumme & $\mathbf{3 , 6}$ \\
\hline
\end{tabular}

fand sich kein Abnehmer (Göschl 2006). Metalle aus Deponien waren oft von niederer Qualität und die Vergütung lag beispielsweise im Bereich von 0-150€/t oder gar im Zuzahlungsbereich (Wiemer 2008). Bei Kunststoffen erschweren starke Verschmutzungen und unbekannte Zusammensetzung die stoffliche Verwertung und auch deren Verwendung als Ersatzbrennstoff (EBS). Glas wurde ebenfalls selten stofflich verwertet und in den meisten Fällen gemeinsam mit der Mineralik entsorgt oder verwertet. Entscheidend für die Verwertung sind aber auch regionale Gegebenheiten und Infrastrukturen, Gewohnheiten von Entsorgungsbetrieben sowie deren individuelle Verträge mit Abnehmern wie beispielsweise mit der Zementindustrie.

Die Erlöse der gewonnenen Wertstoffe lagen bei den bayerischen Deponierückbauten weit unterhalb der Rückbaukosten. Die gegenwärtigen Verwertungswege einzelner Stoffgruppen von Deponierückbauten sind in Tabelle 5 dargestellt.

Für die Bewertung der Energiebilanz sind die Stoffstromwege ausschlaggebend. Bei den bayerischen Rückbauprojekten der letzten Jahre mussten große Mengen Mineralik zu anderen Deponien transportiert werden (Abb. 4). Bei einer Deponie wurde aus den Kunststoffen Ersatzbrennstoff hergestellt. Der Transportweg zwischen Deponie und Verbraucher belief sich auf ca. $250 \mathrm{~km}$ (einfache Richtung).

Hinzu kommt der Energieaufwand für Ausgrabung und Trennung. In Tabelle 6 soll beispielhaft für eine Deponie der Verbrauch für Ausgrabung, Vorsortierung und Transport abgeschätzt werden. Nicht enthalten in der Aufstellung sind 20t Metalle, 20 t Reifen und $10 \mathrm{t} \mathrm{Holz}$, für welche die Verwertungswege nicht bekannt sind. Des Weiteren fehlt der Energieaufwand der Sortierung und des Wiedereinbaus. Der Dieselverbrauch lag bei 25.4951 für $7.080 \mathrm{t}$ Deponat, das entspricht 3,6l/t (ca. 35,3kWh/t bei einem Energieäquivalent von 9,8kWh). Dieser Wert kann sich nahezu verdoppeln, sofern die Rückfahrten der LKWs und der Energieverbrauch der Bodenbehandlungsanlage hinzugerechnet werden. Rettenberger (2009) setzt einen Energiebedarf von $31,3 \mathrm{kWh} / \mathrm{t}$ für das Abgraben, den Transport und die Aufbereitungsanlage an.

Aus den Kunststoffen wurden in einer Müllverbrennungsanlage ca. 80 MW Strom abgegeben, das entspricht $200 \mathrm{kWh} / \mathrm{t}$. Ein Anschluss an das Fernwärmenetz oder ähnliches war nicht vorhanden. Rettenberger (2009) gibt für das Potenzial der Energieerzeugung einen Bereich von 170 


\begin{tabular}{|c|c|c|c|c|c|}
\hline Deponie & Menge & Einheit & Kosten in $€$ & $€ / \mathrm{m}^{3}$ & Anmerkungen \\
\hline Jagerbergl'/Deutschland & 13.000 & $\mathrm{~m}^{3}$ & 3.000 .000 & 230,77 & Kompletter Rückbau \\
\hline Tiefenbach $1 /$ Deutschland & 5.000 & $\mathrm{~m}^{3}$ & 1.120 .000 & 224,00 & Kompletter Rückbau \\
\hline $\begin{array}{l}\text { Holzg'wandenweg'/ } \\
\text { Deutschland }\end{array}$ & 4.150 & $m^{3}$ & 750.000 & 180,72 & Kompletter Rückbau \\
\hline Naples/USA ${ }^{2}$ & 292 & $\mathrm{t}$ & 25.000 & 73,00 & $\begin{array}{l}\text { Pilotversuch; Umrechnungskurs: } 1 \text { USD : } 10,4 \text { ATS } \\
\text { (Aug. 1992); Umrechnungsfaktor: } 1 \mathrm{~m}^{3}=1,16 \mathrm{t}\end{array}$ \\
\hline $\begin{array}{l}\text { Am Heckenweiher }{ }^{1} / \\
\text { Deutschland }\end{array}$ & 38.500 & $\mathrm{~m}^{3}$ & 2.750 .000 & 71,43 & Wiedereinbau Vorort \\
\hline Walkmühle ${ }^{1} /$ Deutschland & 5.900 & $\mathrm{~m}^{3}$ & 330.000 & 55,93 & Kompletter Rückbau ohne Trennung \\
\hline Helsingborg/Schweden ${ }^{3}$ & 165.000 & $\mathrm{t}$ & 3.033 .810 & 22,07 & $\begin{array}{l}\text { Hochrechnung auf Basis von Pilotversuch; Umrechnungs- } \\
\text { kurs: } 10 \text { SEK : } 1,05 € ; \text { Umrechnungsfaktor: } 1 \mathrm{~m}^{3}=1,2 \mathrm{t}\end{array}$ \\
\hline Rettenberger/Deutschland ${ }^{4}$ & & & & 13,00 & Wiedereinbau Vorort \\
\hline Rennerod/Deutschland ${ }^{5}$ & 785.000 & $\mathrm{~m}^{3}$ & 6.500 .000 & 8,28 & Ohne Entsorgungskosten \\
\hline Sharjah/UAE ${ }^{6}$ & 6.400 .000 & $\mathrm{~m}^{3}$ & 35.200 .000 & 5,50 & Ohne Entsorgungskosten \\
\hline Edinburg/USA ${ }^{7}$ & 15.000 & $\mathrm{~m}^{3}$ & & 4,25 & Umrechnungskurs: 1 USD : 12 ATS (Mai 1991) \\
\hline
\end{tabular}

bis $500 \mathrm{kWh} / \mathrm{t}$ für Strom und zusätzlich einen Bereich von 270 bis $733 \mathrm{kWh} / \mathrm{t}$ für Wärme an. Die Rückgewinnung von $3.400 t$ Eisen und $870 \mathrm{t}$ Buntmetalle bei der Deponie Elbisgraben in der Schweiz stellte eine Einsparung von ca. 20 Millionen Liter Erdöl dar, welches einen Brennwert von 211,4 GW bei 10,57 kWh/l entspricht (Fitze et al. 2008).

\section{Kosten und Nutzen}

Die Kosten fangen bei den Voruntersuchungen an und schließen mit der Rekultivierung ab. Mehrere Gutachter, Ingenieurbüros, Labore, Entsorger, Transporteure, Lieferanten und mehr können beteiligt sein. Jedoch wirken sich besonders die Transportdistanzen und die Entsorgungskosten für Stoffe im Zuzahlungsbereich auf die Höhe der Kosten aus. Während die Kosten relativ stabil sind, können die Erlöse hingegen je nach Marktsituation stark schwanken. Da ein Rückbau mit einem gewissen Grundaufwand verbunden ist, sind die relativen Kosten $\left(€ / \mathrm{m}^{3}\right)$ betrachtet, große Deponien begünstigt (Tab. 7).

Der Nutzen lässt sich nicht immer monetär ausdrücken. Dadurch ist eine Gegenüberstellung von Kosten und Nutzen nur bedingt möglich. Bei der Deponie Elbisgraben in der Schweiz lag der Wert für gewonnenes Deponievolumen bei 1,1 Mio. Schweizer Franken (ca. 710.000€), hinzu kommen Erlöse für 3.400 Tonnen Eisen und 870 Tonnen Buntmetalle (Fitze et al. 2008).
In Bayern wurden einige Deponien, die in Einzugsgebieten der Trinkwassergewinnung lagen, zurückgebaut. Die Gegenüberstellung der Rückbaukosten und der Trinkwasserförderungsmengen der betroffenen Brunnen oder Quellen gibt einen Größenanhalt zum Vergleich (Tab. 8). Denn sollte das Wasser aufgrund von Sickerwässern kontaminiert werden, müssten entweder Wasser bei anderen Versorgern zugekauft und ein Versorgungsnetz gebaut, oder spezielle Trinkwasseraufbereitungsanlagen installiert werden. Letztere wären mit erheblichen Anschaffungsund Betriebskosten verbunden. Die Erschließung neuer Grundwasserförderungsgebiete ist sehr begrenzt und stellt deshalb selten eine Lösung dar.

Die Nutzen des Rückbaus können sehr unterschiedlich sein wie folgende Liste zeigt:

- Vermeidung von Emissionen

- Ressourcenschonung und Energiegewinnung
- Schaffung von Deponievolumen und Verlängerung der Deponienutzung

- Anpassung der Deponie an aktuelle technische Standards

- Anlegen von Monobereichen

- Vermeidung der Nachsorge

- Erlöse von gewonnenen Wertstoffen

- Ermöglichung weiterer Nutzungen (Bebauung, Grundwassergewinnung etc.)

- Steigerung des Grundstückswertes

- Kommunale Einnahmen durch jährliche Grund- und Gewerbesteuer

\section{Ausblick}

In den letzten 20 Jahren wurden erhebliche Investitionen getätigt um Deponien rückzubauen. Die technischen Entwicklungen in der Recyclingindustrie führten zu einer Verbreitung erschwinglicher und mobiler Aufbereitungsanlagen. Somit konnten auch klein- und mittelständische Unternehmen Erfahrungen sammeln. Die Pilotphase des Deponierückbaus nähert

\section{TABELLE 8}

Rückbaukosten von Deponien und die Trinkwasserförderungsmengen der betroffenen Brunnen (alle in Bayern)

\begin{tabular}{lccc} 
Deponie & $\begin{array}{l}\text { Rückbau- } \\
\text { kosten in } \mathbf{€}\end{array}$ & $\begin{array}{l}\text { Trinkwasser- } \\
\text { förderung } \\
\mathbf{m}^{3} / \mathbf{J a h r}\end{array}$ & $\begin{array}{l}\text { Verkaufspreis des Trinkwassers } \\
\mathbf{2 0 0 9} \text { in netto } \mathbf{€} / \mathbf{m}^{\mathbf{3}}\end{array}$ \\
\hline Holzg'wandenweg & 750.000 & 50.000 & 1,15 \\
\hline Deponie in Bayern & 250.000 & 300.000 & 1,73 \\
\hline Jagerbergl & 3.000 .000 & 380.000 & 1,33 \\
\hline${ }^{*}$ laut Angabe der zuständigen Wasserwarte & & \\
\hline
\end{tabular}


sich seinem Ende. Die Rückkoppelung mit der Maschinenbauindustrie lässt in $\mathrm{Zu}$ kunft Aufbereitungsanlagen erwarten, die mehr den Anforderungen der Deponattrennung entsprechen. In diesem $\mathrm{Zu}$ sammenhang stellt sich die Frage, ob künftig durch die Preise für Wertstoffe der Deponierückbau zur Wertstoffgewinnung wahrscheinlicher wird.

Vom Standpunkt des Umweltschutzes aus betrachtet, besteht weiterer Untersuchungsbedarf zur Wirkung und zum kumulierten Energieaufwand des Deponierückbaus. Abbau, Trennung und Transport sind teilweise sehr energieintensiv und die Verwertungsmöglichkeiten des mineralischen Anteils noch steigerungsfähig.

Soziale Aspekte beinhalten die Frage nach dem Know-how-Zuwachs und der Schaffung qualifizierte Arbeitsplätze durch Deponierückbau.

\section{Zusammenfassung}

Ein 2006 eingerichteter Unterstützungsfond ermöglichte mehrere Hausmüllde- ponien in Bayern zurückzubauen. Aus sehr unterschiedlichen Gründen wurden Deponien zurückgebaut. Tendenziell waren jedoch Grund- und Oberflächenwasserschutz in ländlichen Gebieten und Platzmangel in städtischen Gebieten ausschlaggebend. Die gewonnenen Erfahrungen und Ergebnisse in Bayern werden mit denen internationaler Deponierückbauprojekte verglichen. Im Vordergrund stehen die eingesetzten Trennverfahren, die gewonnenen Wertstoffe, die Stoffströme und die Verwertung sowie die Kosten und die Nutzen. Bei den bayerischen Fallbeispielen erfolgte die Trennung der Deponate meist in folgende Fraktionen:

- kontaminierte mineralische Feinfraktion zur Deponierung auf geeigneten Deponien,

- mineralische Grobfraktion zur Nutzung im Straßen- und Deponiebau,

- Leichtfraktion (Kunststoff, Holz, Textilien, etc.) zur energetischen Verwertung,

- Reifen zur energetischen Verwertung, und
- Metalle zur stofflichen Verwertung

Die Deponien enthielten einen großen Mineralikanteil, der zum Teil weit transportiert werden musste. Die Transportdistanzen und Entsorgungskosten stellten sehr große Kostenfaktoren dar. Die geringen Erlöse aus rückgewonnenen Wertstoffen machten eine Gegenüberstellung der Kosten mit dem erzielten Nutzen, wie Grundwassergewinnung, sinnvoller. Die Kosten für die Rückbauten betrugen zwischen 250.000 und 3 Mio. Euro, umgerechnet auf einen Kubikmeter waren dies 62 bis 231 Euro. Die Einnahmen für das Trinkwasser aus den betroffenen Einzugsgebieten beliefen sich auf 57.000 bis 519.000 Euro pro Jahr.

\section{Korrespondenz}

Ingo Hölzle

c/o Technische Universität München

Wissenschaftszentrum Weihenstephan

Weihenstephaner Steig 22

D-85350 Freising

E-mail: ingo.hoelzle@mytum.de

\section{LITERATUR}

Bergstedt Erik (2008) Landfill Mining (LFM) - att ta fram begravda resurser. Exempel på planerade/genomförda projekt i Sverige. Avfall Innlandet 2008, Schweden, S 40

Danzer J, Keilhauer M (2008) „Dig and treat“ erste Ergebnisse einer neuen ,Sanierungstechnologie" am Beispiel der ehem. Hausmülldeponie Tiefenbach bei Oberstdorf. 5 .

nie Tiefenbach bei Oberstdorf. 5 . 2008 (Oktober Bodenschutztage Marktredwitz 2008 (Oktober EPA - Environmental Protection Agency of the United States (1993) Evaluation of the Collier County, Florida Landfill Mining Demonstration. Office of Research and Development Washington, DC, EPA/600/R-93/163, S 47

EPA - Environmental Protection Agency of the United States (1997) Landfill Reclamation. Solid Waste and Emergency Response (5306W), EPA530-F-97-001, S 8

Fitze U, Jordi B (2008) Ökologische Optimierung der Abfallverbrennung. In: Bundesamt für Umwelt BAFU (Hrsg), Umwelt, Heft 1/08, S 32-35

Fricke K (2009) Urban Mining - nur ein Modebegriff? In: Müll und Abfall, Ausgabe 10/2009, S 489 GAB - Gesellschaft zur Altlastensanierung in Bayern mbH (2010) Informationsdienst unter www.altlasten-bayern.de

Göschl R (1999) Remediation of disused landfill sites through excavation/redeposition and treatment. Proceedings Sardinia 99, Seventh International Waste Management and Landfill Symposium, S 12
Göschl R (2006) Deponierückbau im Emirat Sharjah. In: Tiefbau, Jg. 118, Nr. 2, 2006, S 66-71 Hogland W, Marques M, Nimmermark S (2004) Landfill mining and waste characterization: a strategy for remediation of contaminated areas. In: Journal of Material Cycles and Waste Management, Volume 6 , Number 2 , September 2004, Springer, S 119-124

Koch K (2005) Remediation of the Old Sharjah Landfill. Zitiert in: Göschl, R.; 2006: Deponierückbau im Emirat Sharjah. In: Tiefbau, Jg. 118, Nr. 2, 2006, S 66-71

Kreisverwaltung des Westerwaldkreises (2006) Den Enkeln werden keine Probleme hinterlassen. Pressemitteilung vom 22.06.2006

Mocker M, Loh I, Franke M, Neddermeyer T, Stenzel F, Faulstich M (2009) Die Deponie als Rohstoffquelle der Zukunft. In: in: Faulstich, M. Mocker, M. (Hrsg.) 5. Fachtagung. Verfahren \& Werkstoffe für die Energietechnik, ATZ Entwicklungszentrum, Sulzbach-Rosenberg, 17. und 18.06.2009, S 181-193

Prechthai T, Visvanathan C, Cheimchaisri C (2006) RDF Production Potential of Municipal Solid Waste. The 2nd Joint International Conference on "Sustainable Energy and Environment (SEE 2006)", 2006, Bangkok, Thailand, S 5 Rettenberger G (2002) Deponierückbau als Alternative zur Sanierung? In: Deponietechnik 2002. Hamburger Berichte 18. Dokumentation der 3. Hamburger Abfallwirtschaftstage vom 14.15. Februar 2002. Hamburger Berichte 18, Ver- lag Abfall aktuell, Stuttgart 2001, S 369-380 Rettenberger G (2009) Zukünftige Nutzung der Deponie als Ressourcenquelle. 11. Münsteraner Abfallwirtschaftstage (Februar 2009), S 9

Scheu M, Bhattacharya JK (1997) Reuse of Decomposed Waste. In: Adrian Coad (Hrsg.) Lessons from India in Solid Waste Management. Loughborough: Water, Engineering and Development Centre, S 16-17

UBA - Umweltbundesamt GmbH (2003) Sanierte Altlast N9: Mülldeponie Helene Berger. Internet: http://www.umweltbundesamt.at/umweltschutz/altlasten/altlasteninfo/sanaltlasten niederoesterreich/n9/

Werellagama DRIB, Samarakoon SMKB (2007) Manufacture and Sale of Landfill Compost - A Sri Lankan Case Study. Proceedings of the International Conference on Sustainable Solid Waste Management, 5-7 September 2007, Chennai, India. S 320-326

Wiemer K, Bartsch B, Schmeisky H (2008) Deponien als Rohstofflagerstätten von morgen Ergebnisse einer hessenweiten Untersuchung. In Witzenhausen-Institut (Hrsg.), Neues aus Forschung und Praxis, Bio- und Sekundärrohstoffverwertung IV, S 685-716

Zobel R, Lerch U, Royl M (2010) Kommunale Altdeponie Marquartstein - Erfahrungen mit Rückbau und stofflicher Trennung. Bayerische Abfall- und Deponietage 2010, S 7 